i-DUST 2010, $01003(2011)$

DOI: 10.1051 idust/201101003

(C) Owned by the authors, published by EDP Sciences, 2011

\title{
Seismicity and hydromechanical behavior of a fractured porous rock under a high pressure fluid injection
}

\author{
B. Derode ${ }^{1, a}$, Y. Guglielmi ${ }^{2}$, F. Cappa ${ }^{1}$, S. Gaffet ${ }^{1,3}$, and T. Monfret ${ }^{1}$ \\ 1 GeoAzur (UMR6526), UNS-CNRS-IRD-OCA, 250 rue Albert Einstein, \\ 06560 Sophia-Antipolis, France \\ ${ }^{2}$ GSRC, University of Aix-Marseille 1, 3 place Victor Hugo, 13331 Marseille, France \\ ${ }^{3}$ LSBB-OCA, La Grande Combe, 84400 Rustrel, France
}

\begin{abstract}
We performed high-pressure water injections in boreholes set in a fractured porous limestone at $250 \mathrm{~m}$-depth in the LSBB URL in order to investigate the coupling relationships between induced seismicity and the rock permeability changes. The seismic events and tilts of the free wall of the gallery were monitored at different locations by two 3-component accelerometers and two 2D tiltmeters located from 1.5 to $2 \mathrm{~m}$ from the injection chamber. Changes in fluid pressure and flow rate were simultaneously monitored in the chamber. A damage-induced permeability increase of a factor of 2.2 occurred after a 10 seconds long swarm of complex seismic events caused by a pressure peak injection of $3.5 \times 10^{6} \mathrm{~Pa}$ and a sudden increase in the injected flow rate from 0 to to $5.3 \times 10^{-5} \mathrm{~m}^{3} \cdot \mathrm{s}^{-1}$. The various angular directions observed on both tiltmeters and 3D accelerometric components indicate that the source of the deformation remains complex and evolves over the time of fluid pressure diffusion in the fractures. At the beginning, signals appear dominated by the source geometry, the rock mass boundary and stress conditions. After the damage and during the pressure decay, tangential slipping of the fracture behavior dominates stress relaxation in the medium. Finally, tilt monitoring coupled with seismo-acoustic measurements present a promising way to quantitatively estimate the relationships between the changes in fractured rocks permeability and seismicity induced by fluid pressurization.
\end{abstract}

\section{INTRODUCTION}

Fracture porous rocks exhibit a high scientific interest as well as energy and environmental challenges. The key idea of this research paper is to present some preliminary experimental results aiming at improving the understanding of the coupling between seismic waves and flow mass transport in fractured - porous multi-saturated rocks. It has been observed for years that seismicity and microseismicity may be induced by artificial fluid injections in deep wells [1] and by natural fluid-deformation couplings in volcanoes, active faults [2] and landslides [3]. Thus, induced seismicity can be an important reservoir or landslide management tool in particular to extract information on the physical state of rocks and fluids at depth. Generally, seismic surveys are located relatively far from the seismic source and laboratory experiments cannot integrate large-scale heterogeneities such as fractures. Experiments that simultaneously measure hydraulic, mechanical and seismic responses are rare [4]. Consequently, multiphysics measurements collected during fluid-induced seismicity at an intermediate scale (i.e. mesoscopic-scale) between the laboratory and the rock volume of kilometer size are of great interest towards a better understanding of fractured porous media. In such rocks, important mechanisms occur at the mesoscopic-scale (i.e. on length scales larger than the pore size but still smaller than the seismic wavelength) where fractures exert considerable influence upon the rock physical properties and play

\footnotetext{
a e-mail: derode@geoazur.unice.fr
}

This is an Open Access article distributed under the terms of the Creative Commons Attribution-Noncommercial License 3.0, which permits unrestricted use, distribution, and reproduction in any noncommercial medium, provided the original work is properly cited. 


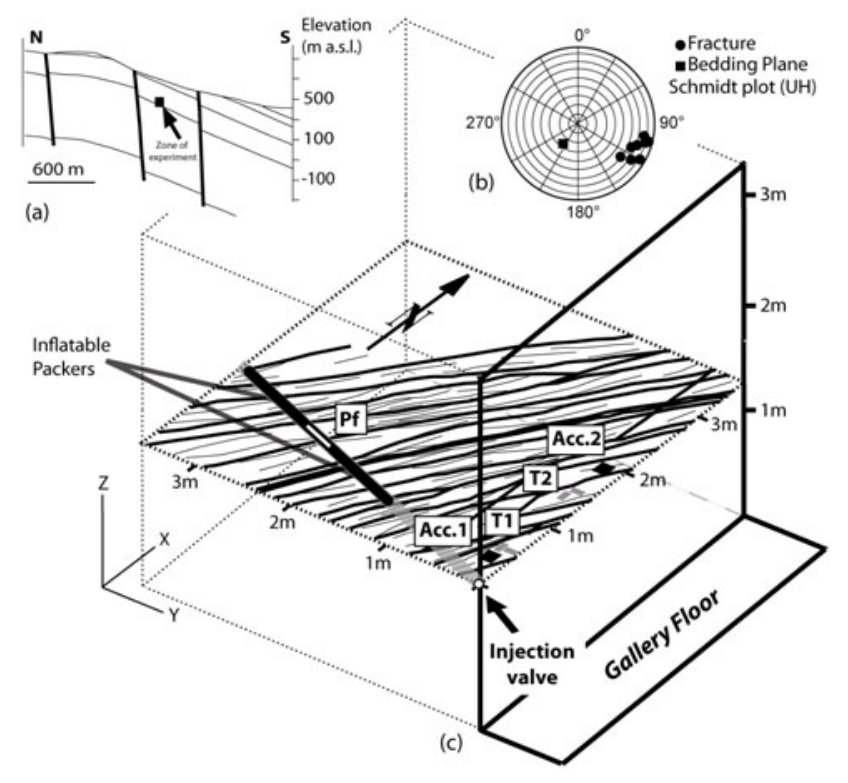

Figure 1. (a) Schematic cross-section of the experiment location in a LSBB gallery in a natural layered carbonate reservoir; (b) Stereographic projection of main fracture planes (upper hemisphere); (c) Detailed geological and experimental set-up (Pf: pressuremeter, Acc: accelerometer, T: tiltmeter).

a key role on both the hydraulic and mechanical behavior of the rock mass [5, 6]. Moreover, despite the fact that these fractures generally represent only small amounts of porosity, they contribute to the difference between static and dynamic responses of rocks because they can vibrate, trap and guide seismic waves, generate resonance $[7,8]$, and conduct fluid flow.

For all those reasons, we developed an in-situ mesoscopic-scale experiment to examine with highresolution the seismicity, the fluid evolution and the mechanical deformations of the porous matrix produced by local and fast (few seconds) high-pressure hydraulic injections in boreholes through a fractured porous limestone of characteristic size of the order of $2 \mathrm{~m}$. In the experiment, fluid injections induced rock damage by producing large changes in effective stress. To our knowledge, the joint analysis of coupled seismic and hydromechanical data presented in this study represents new results in the field.

\section{GEOLOGICAL AND EXPERIMENTAL SETTINGS}

The experiment is set into a gallery wall at $250 \mathrm{~m}$ depth below the ground surface within the Low Noise Underground Laboratory (LSBB) in southern France (Fig. 1a). The LSBB site displays a unique combination of environmental characteristics that lead to a very low-noise geophysical conditions, allowing accurate measurements of small variations in Earth's properties [9]. The tested rock is composed of a layered carbonate with a matrix porosity of 10-15\% intersected by 2 sets of fractures (N30-80W and N120-80NE) with lengths ranging between one to several meters (Fig. 1b-c).

The experiment is a combination of pulses and hydraulic jacking tests conducted in a $0.5 \mathrm{~m}$ screened section of a horizontal borehole $(\varnothing=70 \mathrm{~mm})$ located at $1.5 \mathrm{~m}$-depth perpendicular to the $\mathrm{N} 30^{\circ} \mathrm{E}$ fractures planes (Fig. 1a). During the jacking test the pressure was increased step-by-step until it reached a maximum value of $3.5 \times 10^{6} \mathrm{~Pa}$. Pressure and flow rate measurements were controlled by a water pump located outside of the experiment gallery. Pulse tests were repeated before, immediately after and a few hours after the jacking-test to monitor the changes in the rock mass hydraulic properties related to 
B. Derode et al.: Seismicity and hydromechanical behavior of a fractured porous rock
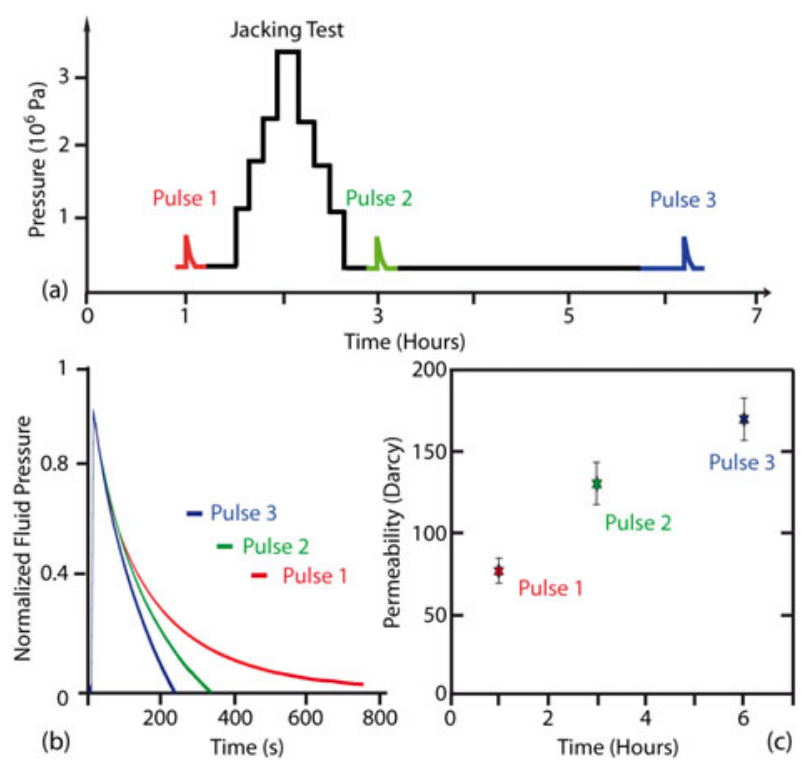

Figure 2. (a) Fluid pressure evolution in the injection chamber during the whole experiment. (b) Normalized fluid pressure for the pulses before end after jacking test.; (c) Intrinsic permeabilities deduced from the pulse tests analysis.

different mechanical states induced by the jacking test. Same experimental protocol was conducted for each of these pulses. The fluid pressure is first increased upstream of a downhole valve to the pressurepulse magnitude of $0.6 \times 10^{6} \mathrm{~Pa}$ by a volumetric water pump. Thereafter, the pulse is initiated when the valve is opened to allow water to enter inside the chamber. The pressure first increases in the chamber and then the valve is closed. After closing the valve, the pressure starts to decrease as a result of fluid flow into the rock mass.

The injection chamber is small enough $\left(\mathrm{V}=2 \times 10^{-3} \mathrm{~m}^{3}\right)$ to avoid any well storage effects. A Keller 26Y pressure sensor with a 0 to 10 bars full-scale allowed accurate measurements of the fluid pressure variations.

Four single axis tiltmeters (Applied Geomechanics, T1 and T2 in Fig. 1b) and two 3-component MEM accelerometers (Colibrys SF3000L, with a flat response in the range of 0.001 to $1 \mathrm{kHz}$ and a $1.2 \mathrm{~V} / \mathrm{g}$ sensitivity) were placed inside small cavities drilled on the gallery wall, at respectively 2.10 and $2.60 \mathrm{~m}$ from the injection chamber (Acc. 1 and 2 in Fig. 1b). Pressure, tiltmeters and accelerometers data were synchronously recorded at a $1 \mathrm{kHz}$ sampling rate without filtering of the instrumental noise.

\section{RESULTS}

The curves for the pressure pulses 1,2 and 3 display different shapes highlighted by different time and slope evolutions for the pressure increasing and decreasing parts.

Indeed, the pressure decreases to reach its initial value in $15 \mathrm{~min}$ for pulse 1 , and only 4 min for pulse 3. Using conventional interpretation methods [9] to derive permeability from the pressure drop, we estimated that this behavior was related to a permeability increase of a factor of 2.2 of the rock mass, respectively from 75 to 170 Darcy (Fig. 2c).

During the jacking-test experiment, pressure is increased during around $400 \mathrm{~s}$ by 5 steps of 50 to $100 \mathrm{kPa}$ (Fig. 3a). Injected flow rate is almost null all along the increasing part of the pressure, which means that the rock mass permeability is low at the initial state. Step-by-step pressure 

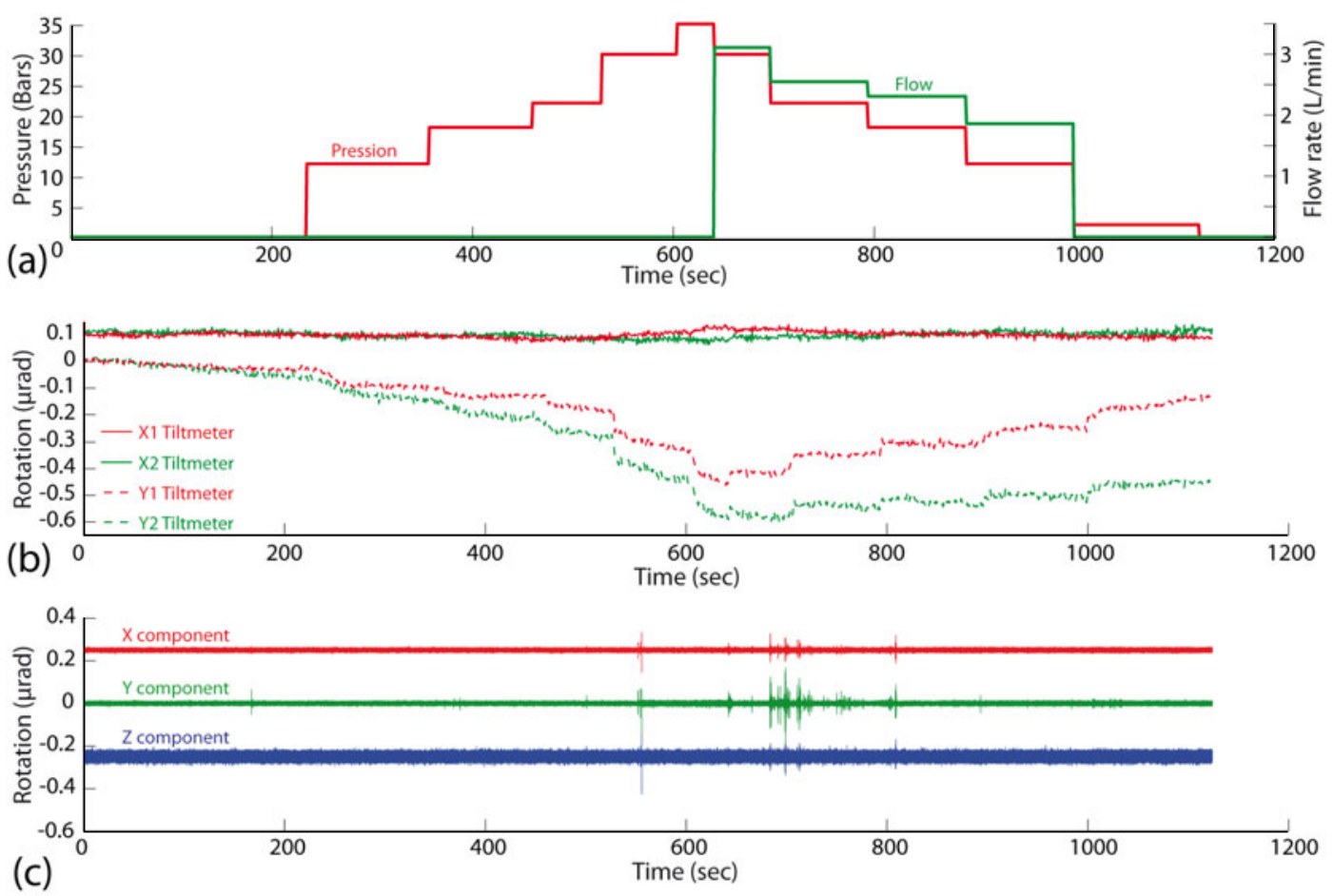

Figure 3. (a) Variations of the pressure (red line) and flow rate (green line) during the jackinc test; ( b) Rotation of the X (full line) and Y (dashed line) tiltmeters during the experiment. Both spatial locations are represented respectively by red and green lines; (c) 3D acceleration measured on the free wall by the near source accelerometer.

increase are clearly seen on all tiltmeters, where tilt magnitudes of -0.5 to $-0.6 \times 10^{-6} \mathrm{rad}$ were measured (Fig. 3b). The changes are mainly observed on the Y-component which is oriented near the normal to the fracture. Thus, the Y-component is well oriented to measure the effect of the fracture opening.

At the maximum injected pressure step, the flow rate suddenly increased to $5.3 \times 10^{-5} \mathrm{~m}^{3} \cdot \mathrm{s}^{-1}$. This sudden inflow was accompanied by a swarm of seismo-acoustic events observed on the three components of both accelerometers, that lasted several seconds. At the end of the pressure decrease, tilt versus time curves displayed a strong hysteresis. This associated with the induced seismicity at the injection peak proves the irreversible damaging of the medium.

The tilt hodographs indicate two different mechanical responses of the medium to the injected pressure (Fig. 4). During the pressure increase, tiltmeter 1 shows a rotation direction which is deviated of $20^{\circ}$ from the normal to the gallery wall during the 3 first pressure steps followed by a rotation direction normal to the gallery wall. Tiltmeter 2 displays a unidirectional rotation normal to the gallery wall during the pressure decrease, and both tiltmeters display a rotation direction which is deviated from the normal to the gallery wall, respectively of $+10^{\circ}$ at tiltmeter 2 and of $-20^{\circ}$ at tiltmeter 1 . The directions of rotation between tiltmeters 1 and 2 clearly show two competing effects on the rotations: (1) the free surface played by the gallery wall, (2) the rock mass pre-existing fractures effect. Indeed, during the pressure increase, the tilt directions appear to be preferentially controlled by the fluid pressure diffusion from a source point influenced by a free to move boundary conditions (the gallery wall). After the irreversible damage of the rock mass, a clear deviation of tilt is observed and can be related to a predominant fractures effect. 
B. Derode et al.: Seismicity and hydromechanical behavior of a fractured porous rock

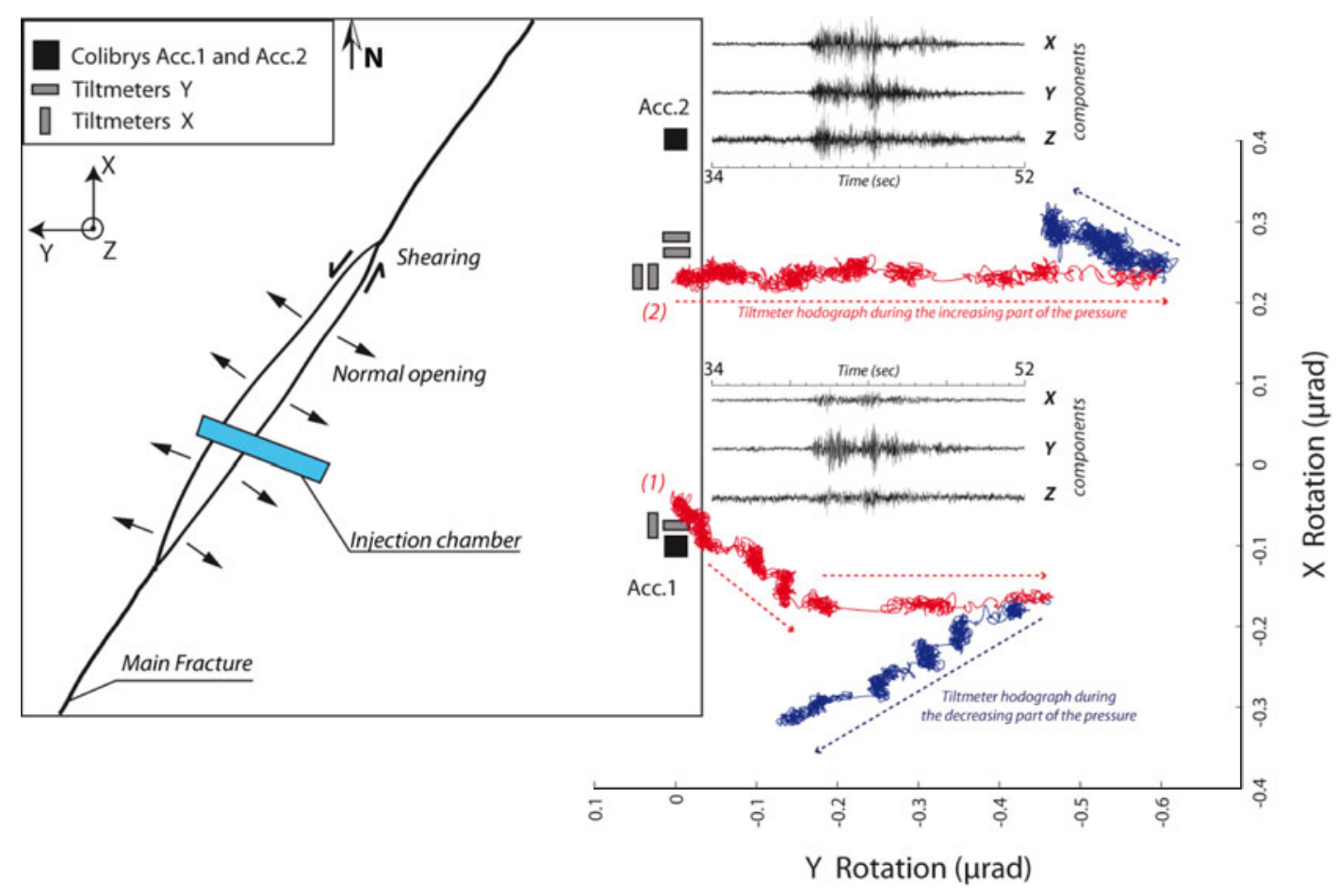

Figure 4. (a) Conceptual scheme of the hydromechanical behavior inside the experiment rock volume. (b) Red curves represent the tiltmeters hodographs during the pressure increasing part of jacking test, and blue curves during the decreasing pressure part. Both are localized at their real special place. Black curves represent the 3D accelerometric data recorded by the Acc. 1 and Acc. 2 Colibrys sensors.

The accelerometric signals recorded at the pressure peak display complex wave trains with different dominant frequencies and polarizations. Although the complete analysis of these signals is not within the aim of this paper, it clearly appears that the X-component magnitude is particularly higher on accelerometer 2 compared to accelerometer 1 . This could be related to the seismic anisotropy of the fractured rock mass, accelerometers 1 and 2 respectively being located in directions perpendicular and almost parallel to the main fracture set.

\section{DISCUSSION AND CONCLUSION}

Our investigation on the hydromechanical behavior a poro-fractured carbonate rock in the near-field of a high pressure injection source indicate a closed correlation between seismicity and inelastic deformation. An increase in permeability of a fractured rock mass by a factor of 2.2 is clearly correlated to seismicity induced by a high-pressure fluid injection in the screened section of a borehole. Static deformation monitored by an array of bidirectional tiltmeters helps understanding the damage processes within a naturally fractured rock. Before damage, the hydromechanical response of the rock is dominated by stress boundary effects while after damage it appears strongly influenced by the preexisting fracture directions. The reason could be that over-pressure imposed to the medium changes the effective stress inside the injected fractures and causes rock bridges breaking inducing heterogeneous 3D movements [10] and associated seismicity. The results is a modification of intrinsic fractures parameters (hydraulic aperture and stiffness) which explains the increase in permeability and the hysteresis seen on the tilt signals. 
This work is funded by the ANR "Captage de $\mathrm{CO}_{2}$ " through the "HPPP- $\mathrm{CO}_{2}$ " project, by the PACA county through the "PETRO-PRO" project, and by the ANR "RiskNat" through the "SLAMS" project. We thank the LSBB engineers team (http://lsbb.oca.eu/) for the technical support during the experiment, and the SITES S.A.S engineer Hervé Caron for their high level technical support during the experiments.

\section{References}

[1] Majer, E.L, Baria, R., Stark, M., Oates, S., Bommer, J., Smith, B., Asanuma, H. (2007), Induced seismicity associated with enhanced geothermal systems. Geothermics, 36, 185-222.

[2] Segall, P. (2009), Earthquake and volcano deformation, Princeton University Press, pp. 432.

[3] Gaffet, S., Y. Guglielmi, F. Cappa, C. Pambrun, T. Monfret, and D. Amitrano (2010), Use of the simultaneaous seismic, GPS and meteorological monitoring for the characterization of a large unstable mountain slope in the southern French Alps, Geophys. J. Int., doi: 10.1111/j.1365246X.2010.04683.X

[4] Guglielmi Y., F. Cappa, and D. Amitrano (2008), High-definition analysis of fluid-induced seismicity related to the mesoscale hydromechanical properties of a fault zone, Geophys. Res. Lett., 35, L06306, doi:10.1029/2007GL033087.

[5] Cappa, F., Guglielmi, Fénart P., Merrien-Soukatchoff V., Thoraval A. (2005), Hydromechanical interaction in a fractured carbonate reservoir inferred from hydraulic and mechanical measurements, Int. J. Rock. Mech. Min. Sci., 42, 287-306.

[6] Cappa, F., Y. Guglielmi, J. Rutqvist, C-F. Tsang, and A. Thoraval (2006), Hydromechanical modeling of pulse tests that measure fluid pressure and fracture normal displacement at the Coaraze Laboratory site, France, Int. J. Rock. Mech. Min. Sci., 43, 1062-1082.

[7] Chouet, B. (1988), Resonance of a fluid-driven crack: radiation properties and implications for the source of long-period events and harmonic tremor, J. Geophys. Res., 93,4375-4400.

[8] Berryman, J.G. (2007), Seismic waves in rocks with fluids and fractures, Geophys. J. Int., 171, 954-974, doi :10.1111/j.1365-246X.2007.03563.x.

[9] Gaffet, S., Y. Guglielmi, J. Virieux, G. Waysand, A. Chwala, R. Stolz, C. Emblanch, M. Auguste, D. Boyer, and A. Cavaillou (2003), Simultaneous Seismic and Magnetic measurements in the LSBB, France during the 2001, 26th January Indian earthquake, Geophys. J. Int., 155, 981-990.

[10] Rutqvist, J., and Stephansson, O. (2003), The role of hydromechanical coupling in fractured rock engineering, Hydrogeology Journal, 11:7-40. 